\title{
Challenges of Medical Home Transformation Reported by 118 Patient-Centered Medical Home (PCMH) Leaders
}

\author{
Leif I. Solberg, MD, A. Lauren Crain, PhD, Juliana O. Tillema, MPA, \\ Patricia L. Fontaine, MD, MS, Robin R. Whitebird, PhD, Thom J. Flottemesch, PhD, \\ Sarab Hudson Scholle, MPH, DrPH, and Benjamin F. Crabtree, PhD
}

Background: Little is known about the most important organizational factors and strategies for transforming primary care clinics into patient-centered medical homes (PCMHs), so we studied this in newly certified medical homes in Minnesota.

Methods: We collected the following information from the first 120 clinics serving adults to be certified: (1) a 105-item survey about the presence and function of practice systems now and 3 years ago; (2) standardized composite clinic performance measures for diabetes and cardiovascular disease; and (3) a 44-item survey about PCMH transformation derived from 31 qualitative interviews about barriers, facilitators, and change strategies with participants from 9 diverse clinics.

Results: The response rates for the systems survey was $\mathbf{9 2 . 5 \%}$ and was $\mathbf{9 8 . 3 \%}$ for the survey about transformation. Nearly all the items from the qualitative interviews identified as potentially important for transformation were strongly endorsed. Eighteen items in this survey also correlated significantly $(P=<.01)$ with change in practice systems at the level of $r \geq 0.20$. However, there was little relationship between these items and either absolute levels of systems or performance on composite measures of diabetes or vascular disease quality outcomes.

Conclusions: Many items in the survey about transformation seem to have face validity for leaders of certified PCMHs and to be associated with the extent to which their clinics have made systems changes. While clinics may need to find their own unique path to transformation, the items identified here should be considered in those decisions. (J Am Board Fam Med 2014;27:449-457.)

Keywords: Medical Home, Organizational Innovation, Patient-Centered Care, Primary Health Care, Quality of Health Care

Although the concept of a medical home for coordinated care of patients with complex medical conditions has been around since at least 1967 , it has attained high visibility and vigorous support only in

This article was externally peer reviewed.

Submitted 25 November 2013; revised 10 March 2014; accepted 20 March 2014.

From the HealthPartners Institute for Education and Research, Minneapolis MN (LIS, ALC, JOT, PLF, RRW, TJF); the National Committee for Quality Assurance, Washington, DC (SHS); and the Department of Family Medicine (BFC), Robert Wood Johnson Medical School, New Brunswick, NJ.

Funding: This project was supported by grant number R18HS019161 from the Agency for Healthcare Research and Quality.

Conflict of interest: none declared.

Disclaimer: The content is solely the responsibility of the authors and does not necessarily represent the official views of the Agency for Healthcare Research and Quality. the past 5 years. ${ }^{1,2}$ This heightened attention has been driven largely by support from both primary care leaders and national policy makers who believe what is now called the patient-centered medical home $(\mathrm{PCMH})$ will rejuvenate essential primary care services while reducing health care costs and improving quality, equity, and patient experience. ${ }^{3-6}$ As of 2010 there were 26 large demonstration projects in 18 states, and many more have begun since then. ${ }^{7}$ This attention also has led to a large number of commentaries, descriptions, and studies in the literature, although until recently few of them addressed the transformation process. ${ }^{8-17}$

Corresponding author: Leif I. Solberg, MD, HealthPartners Institute for Education and Research, PO Box 1524, MS\#21111R, Minneapolis MN 55440-1524 (E-mail: Leif.I.Solberg@HealthPartners.com). 
Although there are now several national and many state and local programs recognizing primary care practices as medical homes, they use a variety of definitions and standards. ${ }^{8}$ Moreover, studies of clinics suggest that transformation is a slow process, with large differences in both clinical processes and outcomes among those that have achieved PCMH status. ${ }^{18-21}$ The transformation process seems to continue even after achieving PCMH recognition. Previous studies have neither identified the maximum level of function and outcomes nor determined what specific actions are needed to most effectively drive transformation.

For those familiar with quality improvement, there should be nothing surprising about the need for ongoing transformation, but this need highlights the importance of identifying the organizational factors that inhibit or facilitate those changes as well as the most effective and efficient strategies for change. Published studies of the PCMH transformation process largely come from the evaluation of the National Demonstration Project, a clustered randomized trial of 36 practices sponsored by the American Academy of Family Physicians in 2006. ${ }^{22}$ Their mixed methods evaluation found that while transformation is feasible, it "requires tremendous effort and motivation, and benefits from external support. Most practices will need additional resources for this magnitude of transformation." ${ }^{23}$ They provided a series of recommendations, most aimed primarily at external sponsors and policy makers. These included the need for disruptive innovations, a systems perspective, and work on building supportive culture, leadership, and teamwork. $^{18,19,23,24}$ Homer and Baron ${ }^{25}$ supported and extended these observations by identifying some critical success factors: leadership, financial resources, personal and organizational relationships, engagement with patients and families, competence in management, improvement methods and coaching, properly applied health information technology, care coordination support, and staff development. A few other qualitative and mixed methods studies focusing on the transformation process in various settings appeared in the past year. ${ }^{11,13,16,17,21,26-28}$ However, few of these studies provide specific factors and strategies needed for success.

Therefore, we sought to supplement these conclusions and recommendations by identifying the specific factors and strategies that differentiated the most transformed medical homes from the least transformed within the first 132 primary care clin- ics in Minnesota to achieve certification as bealth care homes (HCHs), the term used for the $\mathrm{PCMH}$ in Minnesota. Our goal was to assist all primary care clinics in understanding some of the main areas that these transforming clinic leaders believe need particular attention.

\section{Methods}

In 2008, the Minnesota legislature requested the state Departments of Health and Human Services to establish a process for certifying primary medical clinics as HCHs for those wanting to become eligible for special payments for people covered by Medicaid or other state insurance programs. Standards and a certification process were established with community input, and the first clinics were certified in July 2010. Certification standards focused on 5 areas:

1. Continuous access and communication between the $\mathrm{HCH}$ and the patient and family

2. An electronic searchable registry to identify care gaps and manage services

3. Care coordination for patient- and family-centered care

4. Care plans that involve patients with chronic or complex conditions and their families

5. Continuous improvement in experience, health outcomes, and cost-effectiveness

By October 2011, 120 family and/or internal medicine and 12 pediatrics clinics of the $>700$ primary care clinics in the state were certified, and all agreed to participate in this study.

\section{Data Collection}

Practice systems for consistent delivery of medical home services were measured using a questionnaire completed by the lead physician at each clinic. This instrument, the Physician Practice ConnectionResearch Survey (PPC-RS) is a modified version of the survey used by the National Committee for Quality Assurance in its national PCMH recognition program. It has been tested for reliability and validity and demonstrated to be as accurate when completed by the lead physician as when combining various respondents within a clinic. ${ }^{29}$ It contains 105 questions, most of which ask whether a particular system is present and works well, is present but needs improvement, or is not present (eg, 
Does your clinic have a systematic approach to identify and remind patients with chronic illnesses who are due for a follow-up visit?). Following prior methods of PPC-RS scoring, each item receives a $1.0-, 0.5-$, or 0-point score, depending on the answer, and the overall score represents an unweighted percentage of the total possible score of 105 points, resulting in a score ranging from 0 to 100. Respondents were asked to complete each question on the survey twice: once for the current time (2010 to 2011) and again for the same time period 3 years earlier. For ease of interpretation and to focus on systems, we analyzed change in terms of a simple arithmetic difference.

Performance measures for each clinic were obtained from Minnesota Community Measurement, a public reporting organization sponsored by all the health plans in the state. It creates standardized measures that are reported at the individual clinic level on a public website (http://www.mnhealthscores.org). We selected the all-or-none composite measures for vascular disease and diabetes, which combine 4 or 5 separate quality measures into 1 score that represents the proportion of patients with a condition who have accomplished all the measurement goals. ${ }^{30}$ For patients with diabetes, this means each patient needs to have an A1c $<7 \%$, blood pressure $<130 / 80 \mathrm{mmHg}$, low-density lipoprotein $<100$ $\mathrm{mg} / \mathrm{dL}$, and documented nonsmoking and aspirin status. No partial credit is given for attaining less than the full set of goals for each patient. The optimal vascular composite measure is similar except that no A1c level is needed. These data were obtained, when available, for 2008, 2009, and 2010 dates of service so that change over time could be measured during a period similar to that of the systems change.

Potential transformation factors were identified from qualitative interviews of 31 individuals in 9 clinics that were recruited from a group of 10 clinics chosen to reflect diverse organizational types, locations, number of physicians, specialty mix, and performance scores on the practice systems and performance measures. The interviews with physician leaders, administrators, care coordinators, and change leaders at these clinics were designed to identify barriers, facilitators, and strategies that they believed to be most important in transforming into medical homes (not limited to attaining certification). The interviews were audio recorded and transcribed for analysis. Based on the transcripts of these interviews, 5 investigators independently coded key factors (barriers, facilitators, and strategies) identified by respondents as important for transformation and that seemed to differentiate clinics at different levels of achievement. A final coding structure was developed using a constant comparative method and organized the coded data into 7 topic categories of quality improvement, organizational change, culture, leadership, information technology, finances, and patients. The individual items within these categories then were translated into brief statements with 5 response options, ranging from strongly disagree to strongly agree; each statement was scored on a 5 -point Likert scale. The final questions were pilottested with clinic leaders, revised, and sent for electronic completion by the physician and administrative leads at each participating certified clinic in 2012, with regular E-mail and phone follow-up later to attain a high response rate.

Descriptive characteristics were obtained from the questionnaire that was completed by the administrative lead at each clinic. This and all other aspects of research design and methods were reviewed, approved, and monitored by the HealthPartners Institutional Review Board.

\section{Analysis}

Current and previous practice systems scores (PPC-RS) were calculated for each clinic as the proportion of practice systems that were reported to be present and functioning, and the proportions of clinic patients with diabetes or cardiovascular disease who accomplished all measurement goals appropriate for their condition were calculated. Change in practice systems was calculated as the arithmetic difference between current and previous systems scores. Finally, the 3 practice system scores (current, prior, and change) were standardized by the range of scores of all clinics studied to sharpen the focus on each clinic's transformation relative to that of the others.

Responses to negatively worded statements on the survey about transformation were reverse coded so that higher scores always reflected more positively valenced responses. We averaged the responses from each clinic's administrator and physician leader to represent the clinic's score for each question.

Measures of central tendency and dispersion were calculated to describe PPC-RS scores, perfor- 
mance measures, transformation factors, and clinic characteristics in a manner appropriate to the distribution of each variable. Change over time was described as the arithmetic difference between measures, with the significance of that change assessed using a paired samples $t$ test. Spearman rank correlation coefficients quantified bivariate relationships between transformation survey questions and PPC-RS change. In a sample of 100 , a paired samples $t$ test is sufficiently powered to detect mean differences of Cohen's $d \geq 0.283$, and a Pearson correlation coefficient to detect $r \geq 0.275$ (power $=$ $0.80 ; 2$-sided $\alpha=0.05$ ), although a Spearman coefficient may detect smaller relationships in nonnormally distributed data. Rather than rely solely on statistical significance, the threshold of $r \geq 0.20$ was considered practically meaningful and therefore used to identify more important relationships. As such, the interpretation of results focuses on the qualitative distinctions between factors on the survey about transformation that may be associated with systems change rather than the strength of the quantitative relationships.

\section{Results}

The PPC-RS survey was completed by 111 of the 120 adult clinics (92.5\%). Performance measures for 2010 dates of service were available from 114 clinics for diabetes and 116 clinics for vascular disease. Nearly all these clinics had prior years' diabetes and vascular disease data so that change over time could be calculated (109 for diabetes, 106 for vascular disease). The survey about transformation was completed by 107 physician leaders and 113 clinic managers for at least 1 response from 118 clinics $(98.3 \%)$, but in only 101 clinics did both respondents complete the survey (87.2\%).

Table 1 describes these 120 clinics. Nearly all are part of multiclinic systems, 3 of 4 are part of 3 large systems with at least 21 clinics, and most have at least some specialty services in the clinic. All 120 clinics have electronic medical record information systems to some degree and an average of one third of their patients have Medicare or Medicaid insurance.

The mean percentage of potential medical home practice systems was $67.2 \% \pm 14.4 \%$ currently versus $38.5 \% \pm 16.4 \% 3$ years before. The average change in practice systems among the 109 clinics with current and previous scores was both practi-
Table 1. Characteristics of Adult Certified Health Care Homes in Minnesota $(\mathrm{n}=\mathbf{1 2 0})$

\begin{tabular}{|c|c|c|}
\hline Variable & No. & $\% *$ \\
\hline \multicolumn{3}{|l|}{ Location } \\
\hline Metro & 75 & 62.5 \\
\hline Non-metro & 45 & 37.5 \\
\hline \multicolumn{3}{|l|}{ Ownership } \\
\hline Health system & 112 & 93.3 \\
\hline Health plan & 4 & 3.3 \\
\hline Physicians & 2 & 1.7 \\
\hline \multicolumn{3}{|l|}{ Medical services } \\
\hline PC only & 49 & 40.8 \\
\hline PC and some specialty & 14 & 11.7 \\
\hline Multispecialty & 55 & 45.8 \\
\hline \multicolumn{3}{|l|}{ Primary care MDs (n) } \\
\hline $1-3$ & 22 & 18.3 \\
\hline $4-7$ & 47 & 39.2 \\
\hline $8-10$ & 23 & 19.2 \\
\hline$\geq 11$ & 24 & 20.0 \\
\hline \multicolumn{3}{|l|}{ NP/PAs (n) } \\
\hline 0 & 8 & 6.7 \\
\hline $1-3$ & 67 & 55.8 \\
\hline$\geq 4$ & 24 & 20.0 \\
\hline \multicolumn{3}{|c|}{ Clinics in medical group (n) } \\
\hline 1 & 6 & 5.0 \\
\hline $2-4$ & 6 & 5.0 \\
\hline $5-10$ & 11 & 9.2 \\
\hline $11-20$ & 2 & 1.7 \\
\hline$\geq 21$ & 90 & 75.0 \\
\hline \multicolumn{3}{|l|}{ Patient visits/week (n) } \\
\hline$<350$ & 31 & 25.8 \\
\hline $350-550$ & 30 & 25.0 \\
\hline $550-1000$ & 28 & 23.3 \\
\hline$>1000$ & 29 & 24.2 \\
\hline \multicolumn{3}{|l|}{ Patient insurance } \\
\hline Commercial & 116 & $63.6 \pm 22.6$ \\
\hline Medicare & 117 & $17.3 \pm 10.9$ \\
\hline Medicaid & 117 & $14.8 \pm 16.3$ \\
\hline Uninsured & 115 & $3.4 \pm 6.0$ \\
\hline \multicolumn{3}{|l|}{ Medical records } \\
\hline Fully electronic & 110 & 91.7 \\
\hline Paper + electronic & 6 & 5.0 \\
\hline Paper only & 0 & 0 \\
\hline
\end{tabular}

*Data are \% except for Patient insurance, which are presented as mean \pm standard deviation.

MD, medical doctor; NP, nurse practitioner; PA, physician assistant; PC, primary care.

cally and statistically significant $(29.0 \% \pm 16.5 \%$; $P=<.001)$. However, there was large variation across clinics in systems presence at both time points (range, $10-81 \% 3$ years ago to $28-97 \%$ currently) and in the amount of net change ob- 
served within these clinics (range, $-1 \%$ to $+63 \%$ ), including clinics that were part of large groups.

There was a similarly large variation among these certified clinics in their scores on the performance measures. For diabetes control, mean performance was $24.5 \% \pm 8.3 \%$, with a range from $5.9 \%$ to $43.1 \%$, whereas vascular disease control was $41.6 \% \pm 11.5 \%$, with a range from $10.6 \%$ to $63.6 \%$. $+2.3 \% \pm 5.5 \%$ for diabetes (range, $-12 \%$ to $21 \%)$ and $+4.3 \% \pm 7.5 \%$ for vascular disease (range, $-16 \%$ to $27 \%$ ).

Of the 44 items in the survey about transformation, 14 had a mean score of 4 to 4.99 (agree to strongly agree), 25 had scores from 3 to 3.99 (neutral to agree), and only 5 had scores $<3.0$. The mean rating was $3.59 \pm 0.23$. This limited variation reduced our ability to demonstrate correlations between these scores and the other measures, but it confirmed that most of these items were considered to be relevant by clinic leaders. The individual items and their scores are ranked in Table 2.

Few of these items were significantly correlated with current practice systems scores or 2010 performance measures. However, that was not true for change in systems scores over 3 years (the difference between systems score currently and 3 years earlier). In Table 3, survey items about transformation that were correlated with the change in systems at the level of $\geq 0.2$ are listed in order of Spearman correlation coefficient. All but one of the items in the patient category are in this table; the rest come from the categories of culture, organizational change, and finance. Only one is from quality improvement and none are from leadership or informational technology.

\section{Discussion}

We identified 44 specific organizational factors and strategies from interviews in a diverse sample of clinics and using the transformation survey. Among the clinics that have achieved certification as medical homes, nearly all these factors and strategies were endorsed by these medical home leaders as important for transformation. However, few factors or strategies seem to be correlated with measures of either clinic performance or presence of practice systems important for patient-centered care, although nearly half are correlated with clinic change in practice systems over the previous 3 years. The latter finding provides support for the idea that those factors and strategies have some objective importance for transformation as well as having face validity for the responding clinic leaders. We demonstrated in a previous publication that this practice systems measure is associated with changes in performance measures for patients with diabetes or cardiovascular disease. ${ }^{21}$

Many of the items in Tables 2 and 3 were suggested as important by others, usually based on expert opinion. In fact, some of them (patient engagement, care coordinator job description, focus on care plans, use of formal quality improvement techniques) are required by the certification process in Minnesota. This study contributes by (1) empirically identifying these items from those successfully engaged in PCMH transformation, and (2) verifying that some items have a quantitative relationship to transformation, even though we would expect difficulty demonstrating that relationship for individual items, since any changes are complex and multifactorial.

It is also interesting that the survey items that were correlated with systems change were nearly all from the categories of patients, organizational change, and culture. This suggests that clinics that made the greatest changes in their systems were those that paid a lot of attention to the change process, especially regarding their culture and patient-centeredness. Although there were fewer items for the other categories, only 2 (finance and quality improvement) were reported to be important. Our interpretation of the high levels of agreement with most items and their limited relationship to performance measures or absolute numbers of systems is that there are many factors and strategies that are important for transformation, but every clinic is different and may have varying ability to implement them or do so in different ways to address different barriers and changes. It also suggests that there is no small group of strategies that, if implemented, will improve performance measures. This would be in keeping with other findings in the literature. For example, the extensive scientific literature on guideline implementation seems to be finally abandoning its long search for single change strategies in favor of multifaceted ones. Prior et $\mathrm{al}^{31}$ reviewed 33 systematic reviews of guideline implementation strategies and concluded that success required multifaceted strategies. There is also growing recognition that context matters, and a variety of organizational factors affect the direction 
Table 2. Transformation Survey Item Scores among Patient-Centered Medical Home (PCMH) Leaders (n = 118)

\begin{tabular}{|c|c|c|c|}
\hline Item & Category & Mean & SD \\
\hline Providers were well accustomed to the EMR before PCMH. & Information technology & 4.68 & 43 \\
\hline Patient centeredness is a priority for us. & Patients & 4.55 & 0.54 \\
\hline Providing performance results to everyone is important. & QI & 4.42 & 0.53 \\
\hline We want PCMH because it fits our organization's mission. & Culture & 4.39 & 0.69 \\
\hline We have extensive top leadership support for PCMH. & Leadership & 4.31 & 0.56 \\
\hline We worked hard on patient centeredness. & Patients & 4.30 & 0.55 \\
\hline We regularly use QI methods on other projects. & QI & 4.26 & 0.59 \\
\hline We put much effort into making care teams functional. & Organizational change & 4.21 & 0.63 \\
\hline A physician leader to strongly lead change is important. & Leadership & 4.21 & 0.65 \\
\hline PCMH fits our desire to reduce unnecessary care. & Finances & 4.11 & 0.70 \\
\hline Our care teams worked hard on trust and communication. & Organizational change & 4.08 & 0.59 \\
\hline It was worth it to make the change to a PCMH. & Culture & 4.06 & 0.68 \\
\hline We had a specific team to implement PCMH changes. & Organizational change & 4.06 & 0.76 \\
\hline Our larger organization provided support and guidance. & QI & 4.02 & 0.69 \\
\hline It is not critical to have the right person as coordinator.* & Organizational change & 3.99 & 0.69 \\
\hline Creating care plans was a major part of our change. & Organizational change & 3.94 & 0.59 \\
\hline If patients have to pay, they won't enroll in the PCMH. & Finances & 3.91 & 0.70 \\
\hline We have the organizational resources we need. & Leadership & 3.86 & 0.59 \\
\hline We already were doing most of the PCMH activities. & Culture & 3.80 & 0.71 \\
\hline We could obtain needed resources for EMR barriers. & Information technology & 3.78 & 0.71 \\
\hline We used formal QI techniques to develop the PCMH. & QI & 3.68 & 0.76 \\
\hline We are expanding PCMH services to all our patients. & Culture & 3.64 & 0.83 \\
\hline Our PCMH strategy focused on practice system change. & Organizational change & 3.60 & 0.65 \\
\hline Patients report better experiences in our PCMH. & Patients & 3.56 & 0.57 \\
\hline We have a process for using patients as PCMH advisors. & Patients & 3.53 & 0.85 \\
\hline It was not difficult to modify our EMR for the PCMH.* & Information technology & 3.43 & 0.90 \\
\hline MDH leadership was helpful to our PCMH change. & Organizational change & 3.43 & 0.60 \\
\hline Patient partners are part of our change team. & Patients & 3.41 & 0.72 \\
\hline We got input on $\mathrm{PCMH}$ changes from patient partners. & Patients & 3.39 & 0.66 \\
\hline Changing our culture was important to become a PCMH. & Culture & 3.39 & 0.75 \\
\hline We protected clinician income during the change. & Finances & 3.39 & 0.88 \\
\hline The PCMH learning collaborative was helpful. & QI & 3.32 & 0.59 \\
\hline $\mathrm{PCMH}$ reimbursement is a problem. & Finances & 3.28 & 0.72 \\
\hline We still have a long way to go to become a PCMH. & Organizational change & 3.23 & 0.69 \\
\hline The MDH PCMH certification process wasn't burdensome. & Organizational change & 3.17 & 0.74 \\
\hline Care plan development was not difficult.* & Organizational change & 3.13 & 0.79 \\
\hline Buy-in from everyone for the $\mathrm{PCMH}$ was a major challenge. & Culture & 3.11 & 0.83 \\
\hline Public performance reporting wasn't an incentive for us.* & QI & 3.10 & 0.75 \\
\hline Staff job satisfaction has increased with PCMH changes. & Culture & 3.00 & 0.56 \\
\hline Physician satisfaction has increased with PCMH changes. & Culture & 2.89 & 0.67 \\
\hline Few workflow changes were needed.* & Organizational change & 2.85 & 0.77 \\
\hline Financial resources were adequate for added PCMH staff. & Finances & 2.59 & 0.77 \\
\hline Care coordination fee income was a motivator for $\mathrm{PCMH}$. & Finances & 2.50 & 0.76 \\
\hline A care coordination job description isn't important.* & Organizational change & 1.87 & 0.67 \\
\hline
\end{tabular}

*Item is negatively worded and reverse-coded.

EMR, electronic medical record; MDH, Minnesota Department of Health; SD, standard deviation; QI, quality improvement.

and the chances of any change intervention succeeding. ${ }^{32}$ Powell et $\mathrm{al}^{33}$ reviewed 205 sources for disseminating and implementing evidence-based treatments and identified 68 implementation strat- egies that should be combined in multifaceted, multilevel plans tailored to local context.

In an earlier study, leaders of care systems and clinics successfully implementing guidelines con- 
Table 3. Transformation Survey Items Correlated with System Change Score Over 3 Years $(r \geq 0.20)$

\begin{tabular}{|c|c|c|c|}
\hline Transformation Category and Items & Mean & Spearman $r$ & $P$ Value \\
\hline \multicolumn{4}{|l|}{ Organizational change* } \\
\hline Our care teams worked hard on trust and communication. & 4.08 & 0.32 & $<.001$ \\
\hline We had a specific team to implement PCMH changes. & 4.06 & 0.36 & $<.001$ \\
\hline Creating care plans was a major part of our change. & 3.94 & 0.33 & $<.001$ \\
\hline Our PCMH strategy focused on practice system change. & 3.60 & 0.25 & .01 \\
\hline MDH leadership was helpful to our PCMH change. & 3.43 & 0.36 & $<.001$ \\
\hline A care coordination job description isn't important. & 1.87 & -0.40 & $<.001$ \\
\hline \multicolumn{4}{|l|}{ Patients $^{\dagger}$} \\
\hline Patients report better experiences in our PCMH. & 3.56 & 0.29 & .003 \\
\hline We have a process for using patients as PCMH advisors. & 3.53 & 0.34 & $<.001$ \\
\hline We got input on PCMH changes from patient partners. & 3.39 & 0.28 & .004 \\
\hline Patient partners are part of our change team. & 3.41 & 0.34 & $<.001$ \\
\hline \multicolumn{4}{|l|}{ Culture $^{\ddagger}$} \\
\hline It was worth it to make the change to medical home. & 4.06 & 0.34 & $<.001$ \\
\hline We are expanding PCMH services to all our patients. & 3.64 & 0.28 & .003 \\
\hline Staff job satisfaction has increased with PCMH changes. & 3.00 & 0.26 & .01 \\
\hline Physician satisfaction has increased with PCMH changes. & 2.89 & 0.28 & .004 \\
\hline \multicolumn{4}{|l|}{ Finances $^{\S}$} \\
\hline PCMH fit our desire to reduce unnecessary care. & 4.11 & 0.30 & .002 \\
\hline We protected clinician income during the change. & 3.39 & 0.26 & .008 \\
\hline \multicolumn{4}{|l|}{ Quality improvement $\|^{\|}$} \\
\hline We used formal quality improvement techniques to develop the PCMH. & 3.68 & 0.46 & $<.001$ \\
\hline
\end{tabular}

MDH, Minnesota Department of Health; PCMH, patient-centered medical home.

Leadership (3 items) and Information Technology (3 items) items were not related to systems change scores at $r \geq 0.20$.

${ }^{*} r \geq 0.20$ for 6 of 12 items.

${ }^{\dagger} r \geq 0.20$ for 4 of 5 items.

${ }^{\ddagger} r \geq 0.20$ for 4 of 8 items.

$s_{r} \geq 0.20$ for 2 of 6 items.

$\|_{r} \geq 0.20$ for 1 of 6 items.

cluded that it is essential to attend to many contextual factors and to use many strategies. ${ }^{34}$ Those leaders identified 87 factors and 25 strategies that were clustered in 6 categories: organizational capabilities for change, infrastructure for implementation, implementation strategies, medical group characteristics, guideline characteristics, and external environment. All 6 categories were considered to be important, key, or essential by the experienced implementers, although variables within a medical group that directly affect its ability to undertake planned change were rated as much more important than either guideline characteristics or the external environment. They concluded that implementation efforts must use multiple strategies that take account of multiple characteristics of the guideline, practice organization, and external environment.

What we have learned here about heterogeneity of medical home transformation should also be reflected in the limitations of this study. The practices that have applied for and achieved certification as HCHs in Minnesota, as described in Table 2, certainly are not typical of the whole country, and may not even be typical for this state. Their performance on quality measures may vary within the group of certified practices, but, on average, it is significantly higher than the average for noncertified clinics (although it was also higher 3 years before being certified. ${ }^{35}$ Therefore, it is possible that these higher-performing clinics chose to be certified based on a longstanding goal of being leaders, with recognition and payment as collateral goals, rather than because the certification process documented the improvement. Only time will tell whether certification leads to an increase in their lead over noncertified clinics. The certification process in Minnesota is rigorous, although some of the many other demonstration projects nationally are rigorous as well. The specific transformation 
questions also are subjective and capture only the impression of the clinic leaders responsible for the changes, so future studies will need to quantify and validate those impressions. Another limitation is the potential for recall bias in reporting practice systems 3 years ago, bias that might also vary by clinic and context. Despite these caveats, the factors endorsed by medical home leaders here should be useful to clinics elsewhere looking for guidance on how to approach the transformation process. Even if each clinic must find its own way to deal with its unique situation, these findings identify many considerations and specific areas to focus attention on that have been strongly endorsed by leaders of a diverse set of clinics. They highlight the importance of an organized quality improvement process; specific components of organizational change, clinic culture, leadership, information technology, and financial resources; and the role of patients.

This perspective on transformation (that every practice is different and requires its own approach to change that uses multiple strategies that fit its own situation) fits well with the conclusions from the evaluators of the National Demonstration Project. They concluded that the "developmental pathways to success vary by practice" and that there need to be local variations in the development and implementation of the PCMH model. ${ }^{23}$ Despite the hundreds of published articles about the medical home, there is a surprising dearth of even descriptive information about how anyone built one or recommendations about how to do so. There are plenty of articles about the multiple visions of what a medical home should look like, about what is needed to foster the change from the outside, and even a few preliminary studies of effects. If there is no single best path forward, perhaps that dearth of process prescriptions is both understandable and desirable. But it does require individual clinics to assess carefully their own situation and identify those changes and strategies best suited to their situation and context. Perhaps we should all be more humble about our ability to know just what changes are needed in individual clinics and care systems and how others should go about making them. There may not be any silver bullets that will work for all or even most clinics.

The information and analyses presented in this article would not have been possible without the active cooperation of the medical and administrative leaders of the 120 certified medical homes in
Minnesota. The authors are especially indebted to the leaders and other personnel at the 9 clinics that completed the interviews from which the items in the transformation survey were created: CentraCare Clinic-Becker; Essentia Health-Duluth Clinic-Pediatrics; Fairview Clinics-Maple Grove; HealthPartners-St. Paul Clinic; Lakewood Health System-Staples Clinic; Mayo Family ClinicNorthwest; Park Nicollet Clinic-St. Louis Park; United Hospital District-Blue Earth Clinic; University of Minnesota PhysiciansPhalen Village Clinic.

\section{References}

1. Medical Home Initiatives for Children With Special Needs Project Advisory Committee, American Academy of Pediatrics. The medical home. Pediatrics 2002;110(1 Pt 1):184-6.

2. American Academy of Family Physicians, American Academy of Pediatrics, American College of Physicians, American Osteopathic Association. Joint principles of the patient-centered medical home. Washington, DC: Patient-Centered Primary Care Collaborative; 2007. Available from: http://www.aafp.org/dam/AAFP/ documents/practice_management/pcmh/initiatives/ PCMHJoint.pdf. Accessed May 13, 2014.

3. Starfield B, Shi L. The medical home, access to care, and insurance: a review of evidence. Pediatrics 2004; 113(5 Suppl):1493-8.

4. Rosenthal TC. The medical home: growing evidence to support a new approach to primary care. J Am Board Fam Med 2008;21:427-40.

5. Milstein A, Gilbertson E. American medical home runs. Health Aff (Millwood) 2009;28:1317-26.

6. Rittenhouse DR, Shortell SM. The patient-centered medical home: will it stand the test of health reform? JAMA 2009;301:2038-40.

7. Bitton A, Martin C, Landon BE. A nationwide survey of patient centered medical home demonstration projects. J Gen Intern Med 2010;25:584-92.

8. Solberg LI. How can we remodel practices into medical homes without a blueprint or a bank account? J Ambul Care Manage 2011;34:3-9.

9. DeVries A, Li CH, Sridhar G, Hummel JR, Breidbart S, Barron JJ. Impact of medical homes on quality, healthcare utilization, and costs. Am J Manag Care 2012;18:534-44.

10. Peikes D, Zutshi A, Genevro J, Smith K, Parchman M, Meyers D. Early evidence on the patient-centered medical home. Final report. Rockville, MD: Agency for Healthcare Research and Quality; 2012.

11. Calman NS, Hauser D, Weiss L, et al. Becoming a patient-centered medical home: a 9-year transition for a network of federally qualified health centers. Ann Fam Med 2013;11(Suppl 1):S68-73.

12. Donahue KE, Halladay JR, Wise A, et al. Facilitators of transforming primary care: a look under the hood at practice leadership. Ann Fam Med 2013;11(Suppl 1):S27-33.

13. McAllister JW, Cooley WC, Van Cleave J, Boudreau AA, Kuhlthau K. Medical home transformation 
in pediatric primary care-what drives change? Ann Fam Med 2013;11(Suppl 1):S90-8.

14. McMullen CK, Schneider J, Firemark A, Davis J, Spofford M. Cultivating engaged leadership through a learning collaborative: lessons from primary care renewal in Oregon Safety Net clinics. Ann Fam Med 2013;11(Suppl 1):S34-40.

15. McNellis RJ, Genevro JL, Meyers DS. Lessons learned from the study of primary care transformation. Ann Fam Med 2013;11(Suppl 1):S1-5.

16. Rittenhouse DR, Schmidt L, Wu K, Wiley J. Contrasting trajectories of change in primary care clinics: lessons from New Orleans Safety Net. Ann Fam Med 2013;11(Suppl 1):S60-7.

17. Scholle SH, Asche SE, Morton S, Solberg LI, Tirodkar MA, Jaén CR. Support and strategies for change among small patient-centered medical home practices. Ann Fam Med 2013;11(Suppl 1):S6-13.

18. Miller WL, Crabtree BF, Nutting PA, Stange KC, Jaen CR. Primary care practice development: a relationship-centered approach. Ann Fam Med 2010; 8(Suppl 1):S68-79.

19. Nutting PA, Crabtree BF, Miller WL, Stange KC, Stewart E, Jaen C. Transforming physician practices to patient-centered medical homes: lessons from the national demonstration project. Health Aff (Millwood) 2011;30:439-45.

20. Solberg LI, Asche SE, Fontaine P, Flottemesch TJ, Anderson LH. Trends in quality during medical home transformation. Ann Fam Med 2011;9:515-21.

21. Solberg LI, Crain AL, Tillema J, Scholle SH, Fontaine P, Whitebird R. Medical home transformation: a gradual process and a continuum of attainment. Ann Fam Med 2013;11(Suppl 1):S108-14.

22. Nutting PA, Miller WL, Crabtree BF, Jaen CR, Stewart EE, Stange KC. Initial lessons from the first national demonstration project on practice transformation to a patient-centered medical home. Ann Fam Med 2009;7:254-60.

23. Crabtree BF, Nutting PA, Miller WL, Stange KC, Stewart EE, Jaen CR. Summary of the National Demonstration Project and recommendations for the patient-centered medical home. Ann Fam Med 2010;8(Suppl 1):S80-90.

24. Nutting PA, Crabtree BF, Miller WL, Stewart EE, Stange KC, Jaen CR. Journey to the patient-cen- tered medical home: a qualitative analysis of the experiences of practices in the National Demonstration Project. Ann Fam Med 2010;8(Suppl 1):S45-56.

25. Homer CJ, Baron RJ. How to scale up primary care transformation: what we know and what we need to know? J Gen Intern Med 2010;25:625-9.

26. Bitton A, Schwartz GR, Stewart EE, et al. Off the hamster wheel? Qualitative evaluation of a paymentlinked patient-centered medical home (PCMH) pilot. Milbank Q 2012;90:484-515.

27. Gabbay RA, Friedberg MW, Miller-Day M, Cronholm PF, Adelman A, Schneider EC. A positive deviance approach to understanding key features to improving diabetes care in the medical home. Ann Fam Med 2013;11(Suppl 1):S99-107.

28. Han E, Hudson Scholle S, Morton S, Bechtel C, Kessler R. Survey shows that fewer than a third of patient-centered medical home practices engage patients in quality improvement. Health Aff (Millwood) 2013;32:368-75.

29. Scholle SH, Pawlson LG, et al. Measuring practice systems for chronic illness care: accuracy of selfreports from clinical personnel. Jt Comm J Qual Patient Saf 2008;34:407-16.

30. Nolan T, Berwick DM. All-or-none measurement raises the bar on performance. JAMA 2006;295: 1168-70.

31. Prior M, Guerin M, Grimmer-Somers K. The effectiveness of clinical guideline implementation strategies-a synthesis of systematic review findings. J Eval Clin Pract 2008;14:888-97.

32. Marchionni C, Ritchie J. Organizational factors that support the implementation of a nursing best practice guideline. J Nurs Manag 2008;16:266-74.

33. Powell BJ, McMillen JC, Proctor EK, et al. A compilation of strategies for implementing clinical innovations in health and mental health. Med Care Res Rev 2012;69:123-57.

34. Solberg LI, Brekke ML, Fazio CJ, et al. Lessons from experienced guideline implementers: attend to many factors and use multiple strategies. Jt Comm J Qual Improv 2000;26:171-88.

35. Solberg LI, Crain AL, Tillema J, Hudson Scholle S, Fontaine P, Whitebird RR. Medical home transformation: a gradual process and a continuum of attainment. Ann Fam Med. 2013;11(Suppl 1):S108-14. 